Wilfrid Laurier University

Scholars Commons @ Laurier

Physics and Computer Science Faculty

Publications

Physics and Computer Science

1989

\title{
Chaos in a Parametrically Damped Pendulum
}

H.J.T. Smith

University of Waterloo

James A. Blackburn

Wilfrid Laurier University, jabjabjab@cogeco.ca

Follow this and additional works at: https://scholars.wlu.ca/phys_faculty

\section{Recommended Citation}

Smith, H.J.T. and Blackburn, James A., "Chaos in a Parametrically Damped Pendulum" (1989). Physics and Computer Science Faculty Publications. 47.

https://scholars.wlu.ca/phys_faculty/47

This Article is brought to you for free and open access by the Physics and Computer Science at Scholars Commons @ Laurier. It has been accepted for inclusion in Physics and Computer Science Faculty Publications by an authorized administrator of Scholars Commons @ Laurier. For more information, please contact scholarscommons@wlu.ca. 


\title{
Chaos in a parametrically damped pendulum
}

\author{
H. J. T. Smith \\ Department of Physics, University of Waterloo, Waterloo, Ontario, Canada \\ James A. Blackburn \\ Department of Physics and Computing, Wilfrid Laurier University, Waterloo, Ontario, Canada
}

(Received 4 May 1989)

\begin{abstract}
Numerical simulations have shown that a parametrically damped, but otherwise undriven pendulum possesses many of the dynamical modes characteristic of a simple driven pendulum, yet with notable differences. Over most of the parameter space only a stationary steady state is possible. A single domain exists within which are situated three distinct subregions of periodic, multiperiodic, or chaotic motion. The periodic orbits generally occur at half the damping modulation frequency. All of these phenomena have been experimentally observed on an actual pendulum in which parametric damping is generated electronically.
\end{abstract}

\section{INTRODUCTION}

The subject of the dynamical properties of damped pendula, so long relegated to undergraduate mechanics courses, has a new currency since the discovery of chaos in this and many other nonlinear systems. ${ }^{1}$ The importance of uncovering and elaborating hitherto unrecognized modes in the driven pendulum has been enhanced by the fact that this particular system is isomorphic to a current-biased Josephson junction, and thus results that apply to a pendulum can serve to predict the temporal response of these superconducting devices. ${ }^{2-6}$

Several variants of the simple pendulum have also received attention in recent years. Beckert et al. ${ }^{7}$ experimentally probed the behavior of a pendulum which had the usual velocity-dependent damping and, in addition, a restoring torque proportional to the angular displacement. In a series of papers, ${ }^{8-1 t}$ Leven et al. considered the motion of a parametrically forced pendulum, that is, a pendulum whose suspension point was subjected to a periodic vertical displacement. Inoue ${ }^{12}$ applied perturbation theory to the problem of an undriven simple pendulum having a velocity- and displacement-dependent damping. Chaos in the more elaborate situation of a pendulum suspended within a rotating frame was treated by Shaw and Wiggins. ${ }^{13}$ Finally, we note the work by Romeiras and $\mathrm{Ott}^{14}$ on strange but nonchaotic attractors for a damped pendulum with quasiperiodic forcing.

We report here studies carried out on a parametrically damped, but otherwise undriven, simple pendulum. Parametric damping is introduced by the addition of a prefactor, which is harmonic in time, to the usual velocity-dependent term in the equation for the pendulum. Computer simulations, and experiments performed on an actual pendulum, were employed to map out the chaotic and periodic motions of this system.

\section{THEORY AND NUMERICAL RESULTS}

The well-known equation of motion of a simple pendulum consisting of a mass $m$ situated at a distance $b$ from a pivot is

$$
I \frac{d^{2} \theta}{d t^{2}}+A \frac{d \theta}{d t}+m g b \sin (\theta)=0,
$$

where $\theta$ is the angular displacement measured clockwise from the rest (down) position, $I$ is the total moment of inertia of all rotating components, $g$ is the acceleration of gravity, and the constant $A$ specifies the strength of velocity-dependent damping. Recent reviews which treat the classical behavior of this system, including the effects of additional types of damping, can be found in Refs. (15) and (16). In this work, we consider the effect of adding a time-dependent term to the damping, such that Eq. (1) becomes

$$
I \frac{d^{2} \theta}{d t^{2}}+[A+B \sin (\omega t)] \frac{d \theta}{d t}+m g b \sin (\theta)=0 .
$$

The undamped natural frequency of the pendulum is given by $\omega_{0}=(m g b / I)^{1 / 2}$. A convenient dimensionless form of (2) is obtained if time is expressed in units of $\omega_{0}^{-1}$; derivatives with respect to this new time will be indicated by overdots. Hence

$$
\ddot{\theta}+Q^{-1}\left[1+\epsilon \sin \left(\Omega t^{*}\right)\right] \dot{\theta}+\sin (\theta)=0,
$$

where $Q=(m g b I)^{1 / 2} / A, \epsilon=B / A$, and $\Omega=\omega / \omega_{0}$. Because most of the experiments (to be described in Sec. III) were carried out with $Q=18.33$, this value was employed throughout the simulations. Numerical solutions of Eq. (3) were obtained with a fourth-order Runge Kutta algorithm. A basic time grid of 100 points per cycle of $\sin \left(\Omega t^{*}\right)$ was employed, with half-step error checking. ${ }^{17}$ 


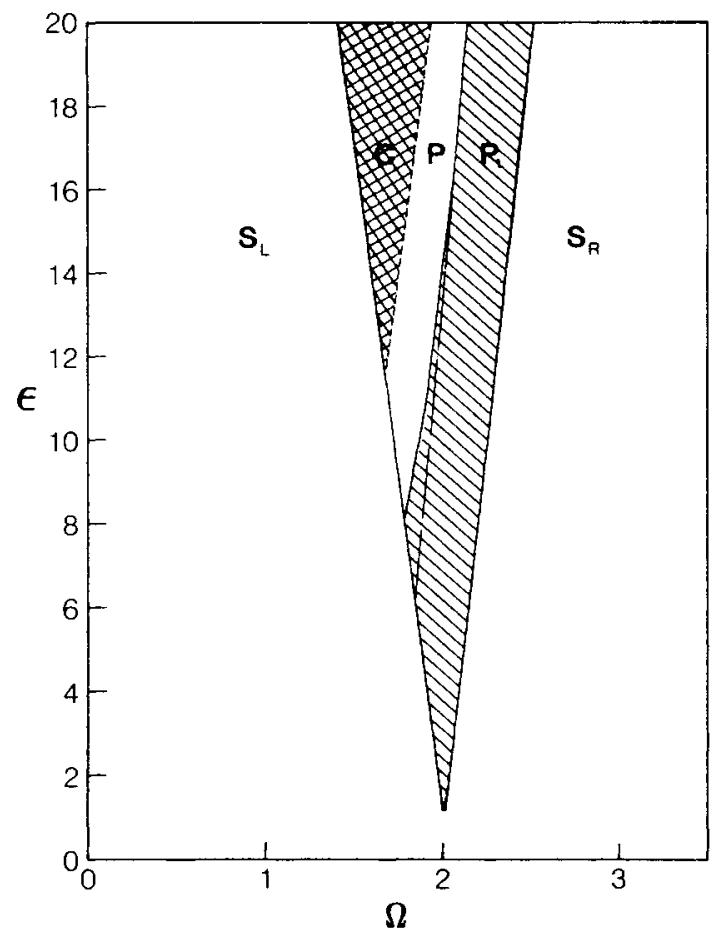

FIG. 1. State diagram for the parametrically damped pendulum. Motion within the regions is as follows: $P_{1}$, period $1 ; P$, multiperiodic; $C$, chaotic. The line drawn within $P_{1}$ is the locus of points for which the oscillation amplitude just reaches $\pi / 2$.

All calculations were performed in double precision arithmetic. It may be noted here that in some cases as many as 10000 such cycles had to be computed to guarantee transient-free solutions, and that this in turn involved something on the order of $3 \times 10^{6}$ passes through the algorithm.

The principal objective of the numerical simulations was to reveal the character of the state space, in other words, to determine the types of motion resulting from given choices of $\epsilon$ and $\Omega$. A discussion of the various modes of the pendulum is probably best served by beginning immediately with the final-state diagram (Fig. 1), which was itself constructed from the many individual computer runs required to delineate the indicated regions.

It is apparent that $\theta=\dot{\theta}=0$ always satisfies Eq. (3); however, as we shall see, such a solution is not necessarily a stable one for arbitrary $(\epsilon, \Omega)$. Nevertheless, simulations revealed that beyond the right-hand boundary of the large $V$ shaped domain (region $S_{R}$ ), this solution always obtains, regardless of the initial conditions $\left(\theta_{0}, \dot{\theta}_{0}\right)$ that are chosen. An example is provided by Fig. 2, which illustrates the decay of oscillations towards a stationary state $(\theta=\dot{\theta}=0)$ in the phase plane, for $\epsilon=16.0$ and $\Omega=5.0$. The circles in this and subsequent figures are markers indicating the beginning of each cycle of $\sin \left(\Omega t^{*}\right)$; in this instance, the damping modulation produces the fivefold undulations which are superimposed on the exponential contraction of the orbit.

$S_{L}$ denotes the portion of the state space that lies to
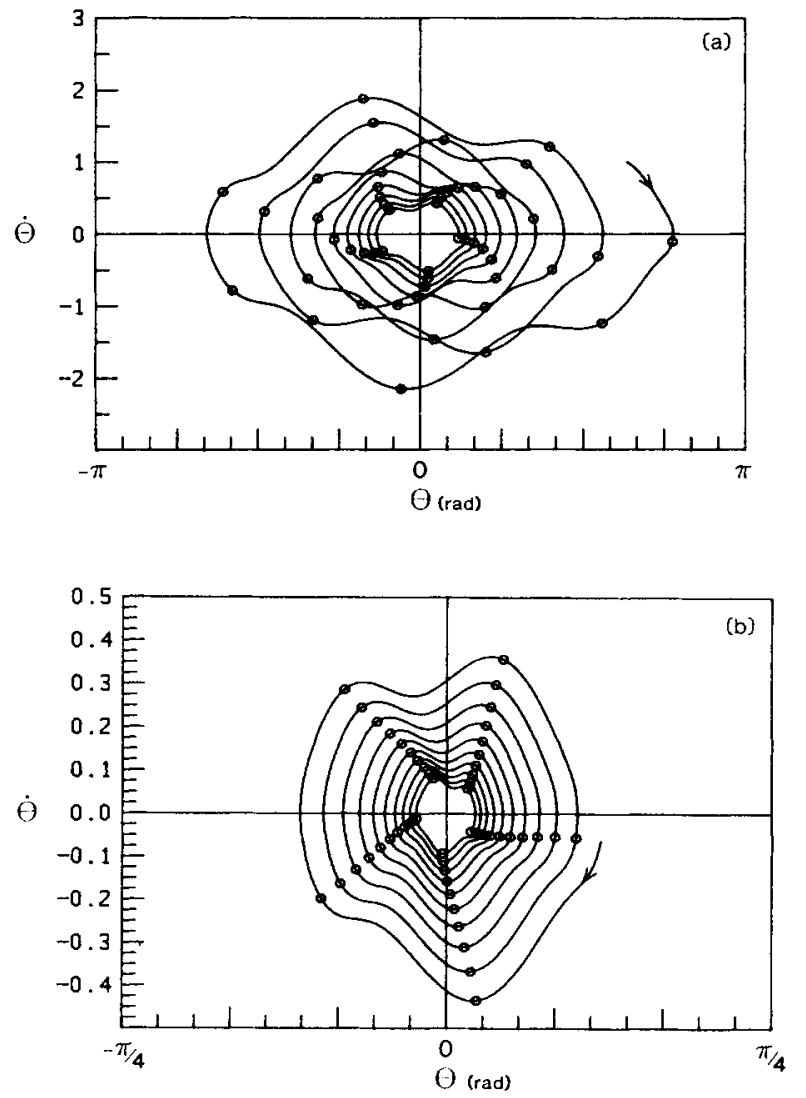

FIG. 2. Decay to a stationary state in the phase plane. $Q=18.33, \epsilon=16.0$, and $\Omega=5.0$. The initial motion (a) is somewhat irregular, but later (b) it becomes synchronized to the damping modulation.

the left of this $V$-shaped region. Most of $S_{L}$ is populated with stationary solutions which are reached after a brief interval of rapidly damped motion. However, very close to the boundary of the subregion labeled $C$, long chaotic transients (lasting as much as several thousand modulation cycles) can precede the ultimate decay to rest. Elsewhere in $S_{L}$, the behavior can be more complex, and other nonstationary attractors may be found. For example, as shown in Fig. 3, suitable parameters $(\epsilon, \Omega)$ and initial

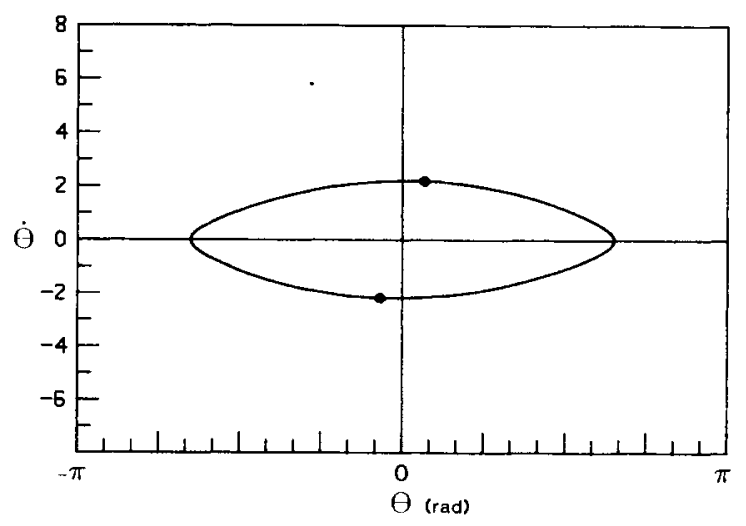

FIG. 3. Phase plane plot of a period-1 solution in region $S_{L}$ at $\epsilon=10.0$ and $\Omega=1.70$ with initial conditions $\theta_{0}=\dot{\theta}_{0}=1.0$. 
conditions can lead to a simple oscillating solution. From the pattern of time markers (circles), it is apparent that the repetition time in this case is two cycles of the modulation period. A slight change in parameter values coupled with new initial conditions leads to the attractor shown in Fig. 4. This is a period-3 solution that requires six drive cycles for completion. A useful visualization of the motion is realized by constructing a polar $t$ plot in which the radius vector is time, measured with respect to some chosen value $t_{0}$ at the origin, and the polar coordinate is the actual pendulum displacement $\theta$. Figure 4(b) presents the trajectory of Fig. $4(\mathrm{a})$ in this alternate manner; the repetitive period-3 pattern is quite evident. Finally, a running solution, which is locked to the damping modulation, is shown in Fig. 5.

It is possible for the attractors mentioned above to coexist at given $(\epsilon, \Omega)$, that is at fixed points in region $S_{L}$ of the state diagram. The choice of initial conditions then determines which of the attractors is selected by the system. This is illustrated by the basin of attraction presented in Fig. 6; it is essentially a map of the outcome of trying all combinations of 143 different $\theta_{0}$ with 101 values of $\dot{\theta}_{0}$ over the ranges indicated. For each of the
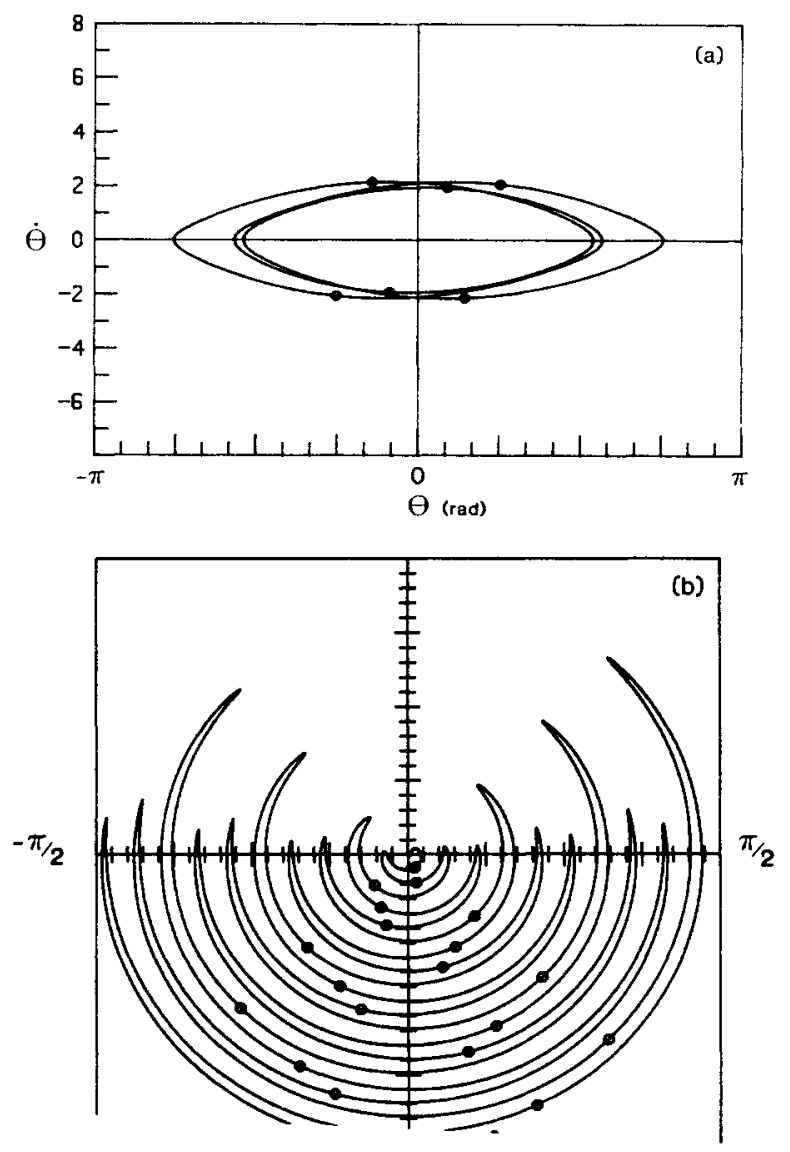

FIG. 4. (a) Phase plane portrait of a period-3 solution in $S_{L}$ at $\epsilon=10.0$ and $\Omega=1.71$ with initial conditions $\theta_{0}=0, \dot{\theta}_{0}=1.0$. (b) Polar $t$ plot of the same motion as depicted in (a). The radius (time) spans 20 modulation cycles.

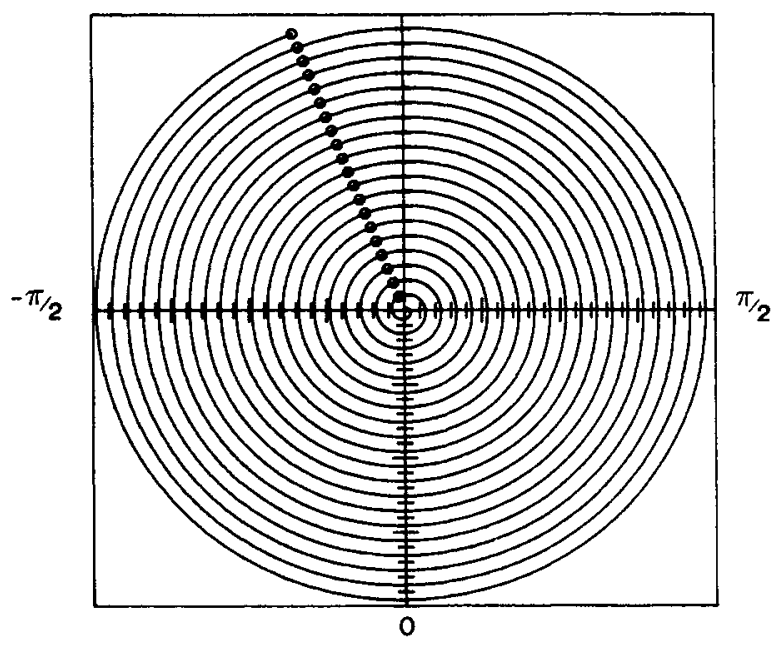

FIG. 5. Polar $t$ plot of running solution in $S_{L}$ at $\epsilon=6.0$ and $\Omega=1.70$. Initial conditions were $\theta_{0}=1.9859155$ and $\dot{\theta}_{0}=0.40$.

14443 initial conditions, Eq. (3) was solved over a total time span covering 55 modulation cycles. The first $50 \mathrm{cy}$ cles were discarded to eliminate transient effects, and the behavior in the remaining five cycles was examined. The figure vividly indicates the complexity of even this nonchaotic motion. Although there is a uniform region surrounding the origin in which a stationary state is ultimately reached, the structure elsewhere appears to be fractal. Fractally intermixed basins have already been observed for the forced pendulum, ${ }^{18,19}$ and for the radiofrequency ( $r f)$-driven Josephson junction. ${ }^{20,21}$

In Fig. 1 regions $S_{L}$ and $S_{R}$ are separated by a wedgeshaped domain within which the stationary state $\theta=\dot{\theta}=0$ appears to be unstable. This is evidenced by the fact that even initial conditions such as $\theta_{0}=\dot{\theta}_{0}=10^{-8}$ lead quickly to oscillating or rotating motion. As shown in the figure, this domain is subdivided into three distinct areas $P_{1}, P$,

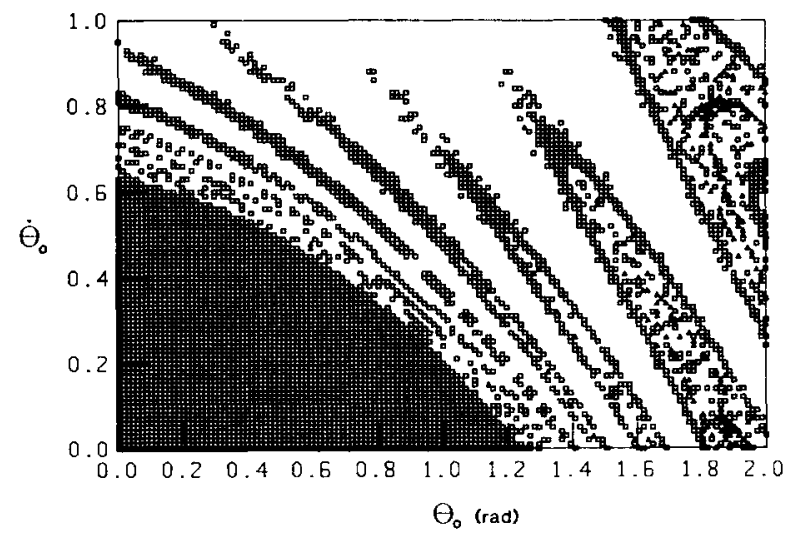

FIG. 6. Basin of attraction for $Q=18.33, \epsilon=6.0$, and $\Omega=1.70$. The computational grid is $143 \times 101$ points. Small squares denote stationary states, while triangles indicate running solutions. Blank areas are periodic, or multiperiodic. 


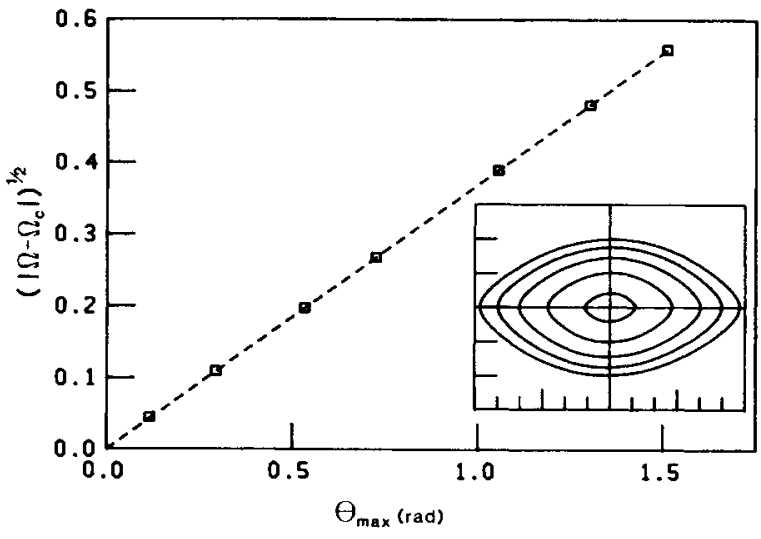

FIG. 7. Maximum $\theta$ for several phase plane orbits within $P_{1}$ as a function of $\left.\left(\mid \Omega-\Omega_{c}\right)\right|^{1 / 2}$ at $\epsilon=16.0$ (for which $\Omega_{c}=2.412$ ). Inset: phase plane plot showing four limit cycles. The horizontal scale is from $-\pi / 2$ to $\pi / 2$.
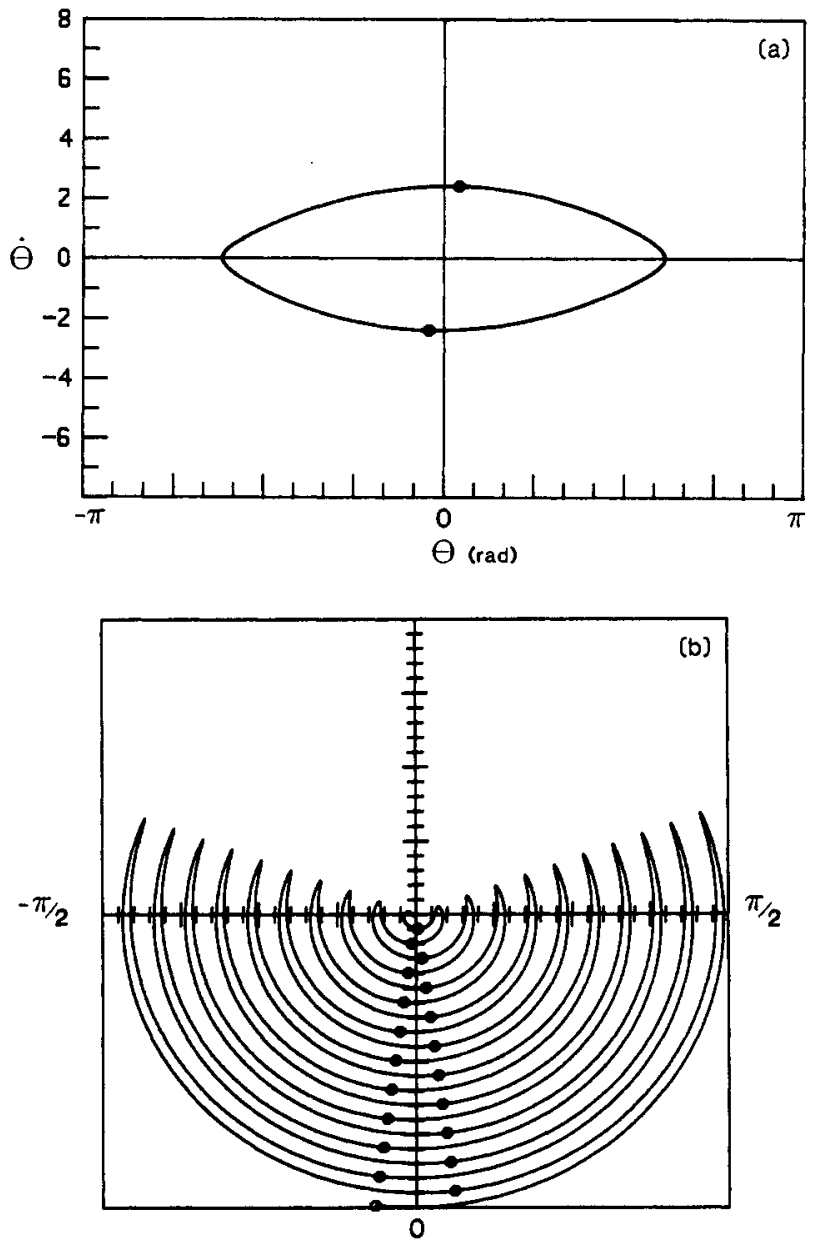

FIG. 8. (a) Phase plane plot in region $P$ with $\epsilon=16.0$ and $\Omega=1.90005$. Initial conditions were $\theta_{0}=\dot{\theta}_{0}=0.001$. (b) polar $t$ plot. and $C$.

Region $P_{1}$. Within $P_{1}$, the steady-state motion of the pendulum is period 1. Each complete oscillation spans two drive cycles of the damping modulation. The amplitude $\theta_{\max }$ of the oscillation is zero along the right-hand boundary of $P_{1}$, and is $\pi / 2$ or slightly larger along the left-hand boundary. This can be seen in the inset of Fig. 7 where limit cycles resulting from four choices of $\Omega$ are plotted. The boundary between $S_{R}$ and $P_{1}$ is a line of Hopf bifurcations ${ }^{1}$ marking the transition from fixed point to limit cycle. When $\epsilon$ is held constant and $\Omega$ is treated as a control parameter, we find that $\theta_{\max }$ across $P_{1}$ scales as $\left(\left|\Omega-\Omega_{c}\right|\right)^{1 / 2}$, where $\Omega_{c}$ is the value of drive frequency at the transition boundary (Fig. 7).

Region $P$. The conditions in this region (large enough $\epsilon$ and/or small enough $\Omega$ ) are such that the maximum pendulum excursions sufficiently exceed $\pi / 2$ to allow rotating states to emerge; $P$ is thus characterized by a mixture of periodic and multiperiodic states. For example, as illustrated in Figs. 8 and 9 , a small shift in $\Omega$ can
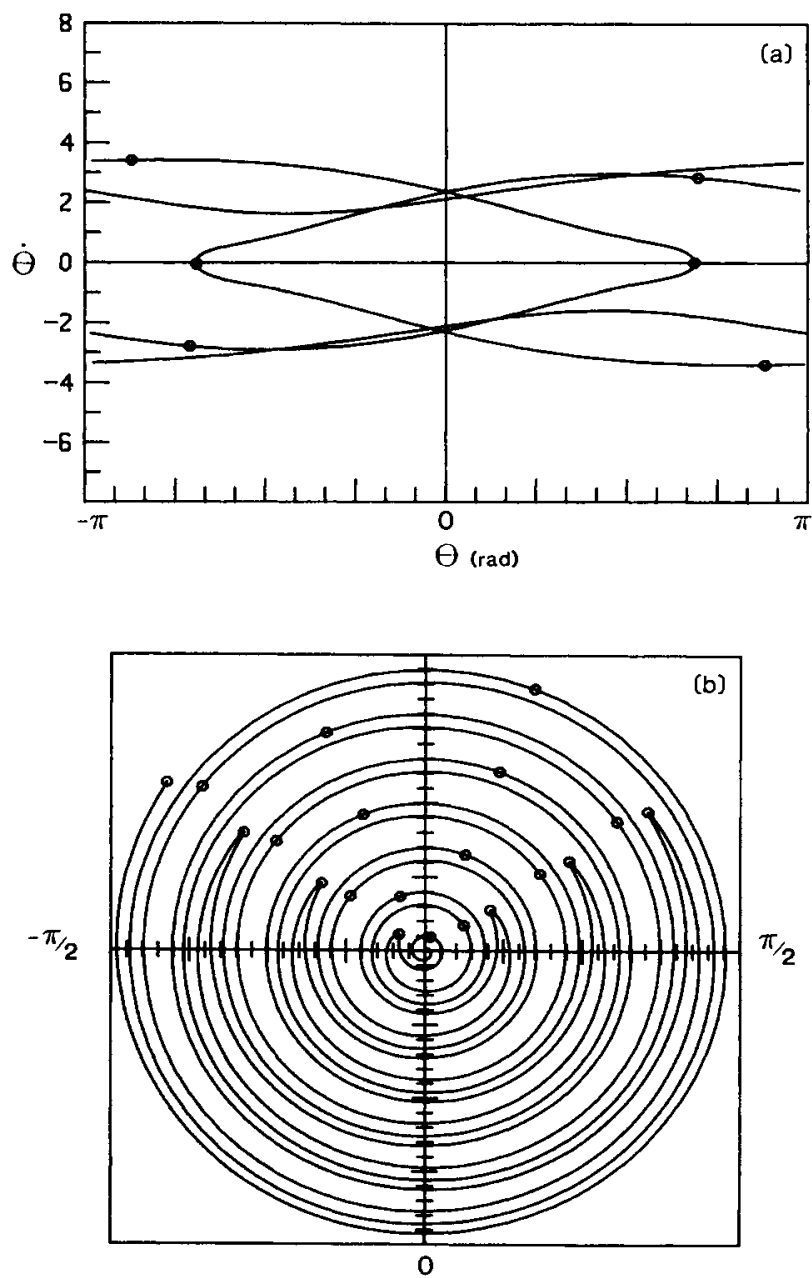

FIG. 9. (a) Phase plane and (b) polar $t$ plots for same conditions as in Fig. 8, except $\Omega=1.90007$. 
change the motion from nonrotating period 1 to rotating period 3.

Region $C$. Chaotic motion is contained within region C. As is typical of such domains, on a fine enough scale it appears to be permeated by nonchaotic zones as well. We have not attempted to map out these structural details. A typical chaotic phase plane orbit is shown in Fig. $10(a)$, while the corresponding polar $t$ plot is presented in Fig. 10(b). Poincaré sections were computed by sampling the numerical solution once in alternate drive cycles. Because of the possibility of nonchaos being masked by long chaotic transients, these plots were constructed from data following a 20000 cycle "settling time." In Figs. 11(a) and $11(\mathrm{c})$ are shown two Poincaré sections obtained from the same chaotic motion, but differing in the phase of the sampling instant with respect to $\sin \left(\Omega t^{*}\right)$. This points out the sensitivity of the general appearance of a Poincaré section to the choice of sampling moment. The magnified portion of the strange attractor illustrated in Fig. 11(b) typifies the self-similar nature of these fractal structures.
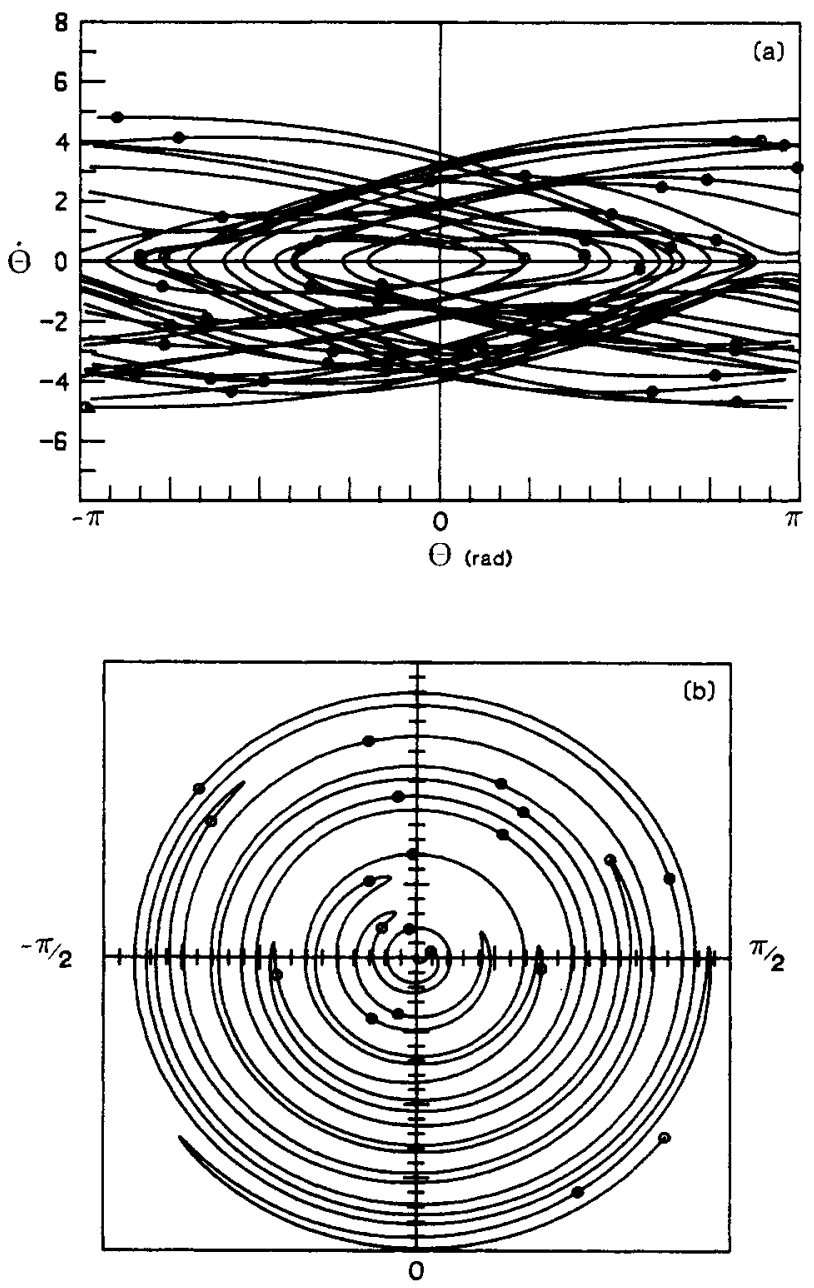

FIG. 10 (a) Phase plane and (b) polar $t$ plots showing chaos for $\epsilon=19.8$ and $\Omega=1.80$.
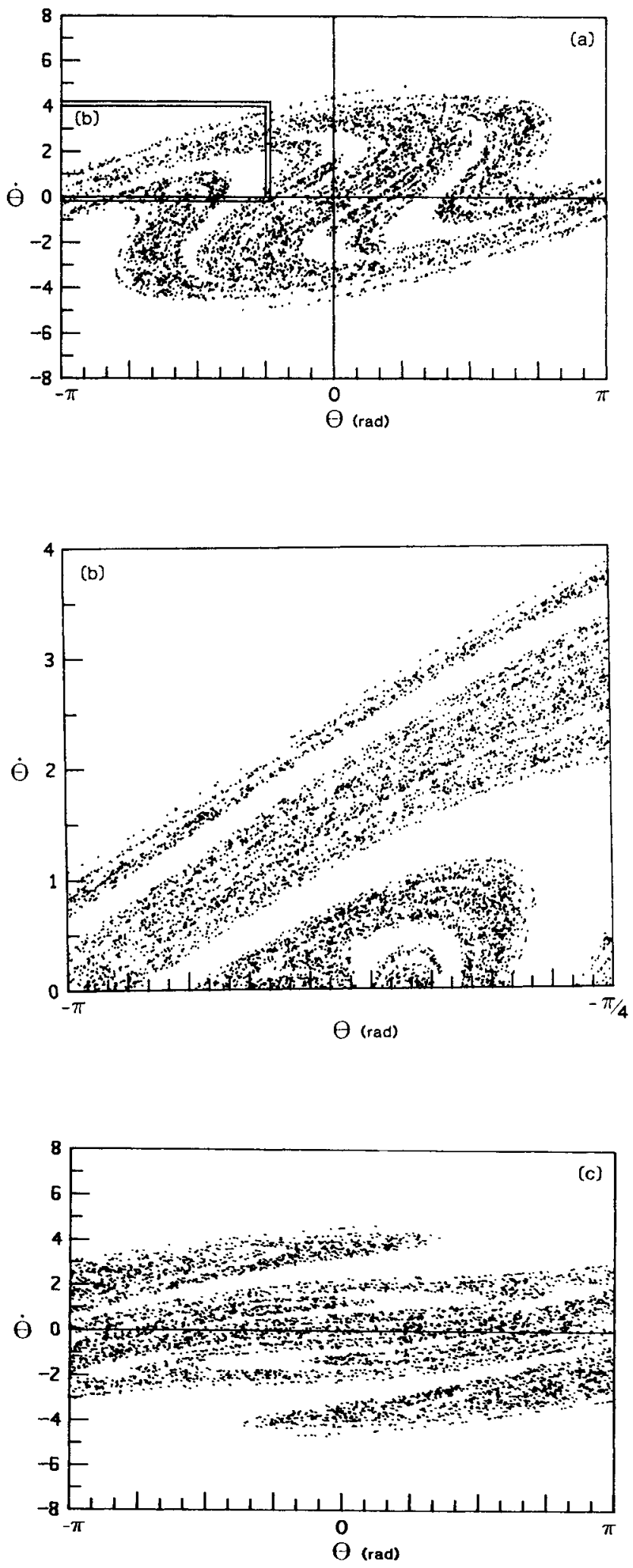

FIG. 11. Poincare sections of the chaotic trajectory shown in Fig. 10: 5000 sampled points are plotted. (a) Sampling phase shift of $27^{\circ}$; (b) boxed region of the strange attractor, magnified; (c) sampling phase shift of $153^{\circ}$. 


\section{EXPERIMENTAL APPARATUS AND RESULTS}

The apparatus consisted of a modified version of the driven pendulum described by Blackburn et al. ${ }^{22}$ Since that reference contains a detailed description of the experimental system, we shall confine ourselves here to a discussion of the modifications, which were required for the present study.

In the original apparatus, torque was induced by a set of four stationary flatwound coreless drive coils, configured as two orthogonal pairs, which were closely coupled to a rotor ring magnet. Damping was generated by eddy currents induced in a fixed copper plate. The new pendulum has no copper plate or micropositioner; damping torques are created electronically as described below.

As in the earlier design, the annular ring magnet had four north and four south poles alternating around its circumference (see Fig. 12). The magnet was fixed coaxially to the pendulum spindle and was positioned very near (and parallel) to the surface of the printed circuit board on which the drive coils were mounted. A new feature, as illustrated in Fig. 12(b), is the addition of a pair of velocity sensing coils. The mechanical angle between them $(\pi / 8)$ means that when rotating, the ring magnet will in-
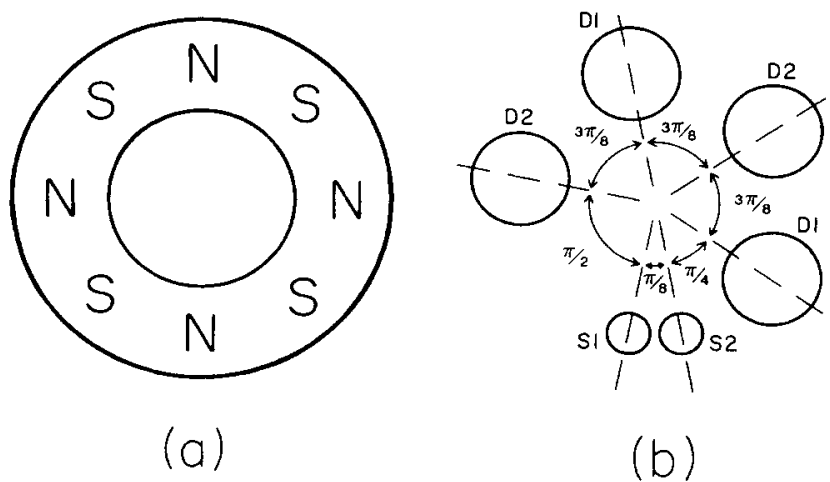

(b)

FIG. 12. (a) Diagram of ring magnet and (b) geometric arrangement of drive coils and velocity sensing coils.

duce coil signals that are $\pi / 2$ electrical degrees apart. Similarly, the mechanical angle of $3 \pi / 8$ between the two $D_{1}$ coils assures that, when energized, they will create a torque on the magnet in a push-pull fashion, and likewise for the $D_{2}$ coils.

From the above remarks, it is clear that if the ring

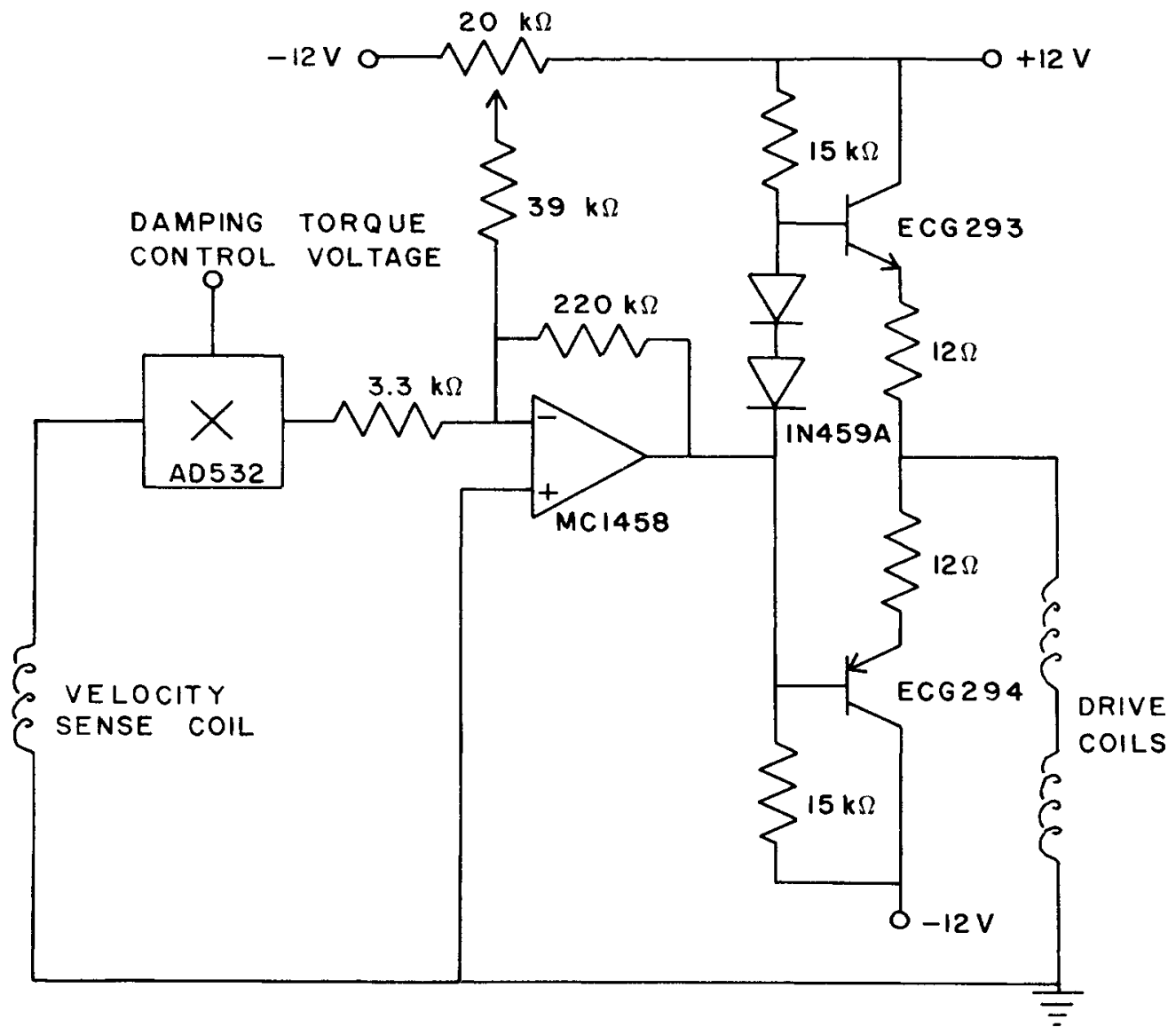

FIG. 13. Schematic diagram (one of two channels) of motor drive circuit. 
magnet generates a field $B_{1} \propto \sin (4 \theta)$ at sense coil 1 , then the field at sense coil 2 will be $B_{2} \propto \sin (4 \theta+\pi / 2)$. As shown in Fig. 13, the signal from coil 1, which is proportional to $d B_{1} / d t$, is multiplied by an externally supplied control voltage $\left(V_{d}\right)$, and the resulting current is fed to drive coils $D_{1}$. The signal from sense coil 2 is processed similarly.

The coils were wound in such away that the net torque generated was proportional to $-B_{1} d B_{2} / d t+B_{2} d B_{1} / d t$ or

$$
\begin{aligned}
\tau \propto V_{d}\left(-4 \sin (4 \theta) \cos (4 \theta+\pi / 2) \frac{d \theta}{d t}\right. \\
\left.+4 \cos (4 \theta) \sin (4 \theta+\pi / 2) \frac{d \theta}{d t}\right] .
\end{aligned}
$$

Hence

$$
\tau=K V_{d} \frac{d \theta}{d t}
$$

where $K$ is determined by circuit components and the magnet to coil coupling. Equation (4) confirms the fact that this new design electronically generates a velocitydependent torque. This technique provides damping which is inherently a linear function of the control voltage; eddy current damping, on the other hand, is nonlinearly dependent on plate-to-magnet separation. ${ }^{22}$

In order to test the precision with which the circuit and coil assembly actually adheres to the damping relationship expressed by Eq. (4), the pendulum was temporarily removed from the apparatus, leaving only the

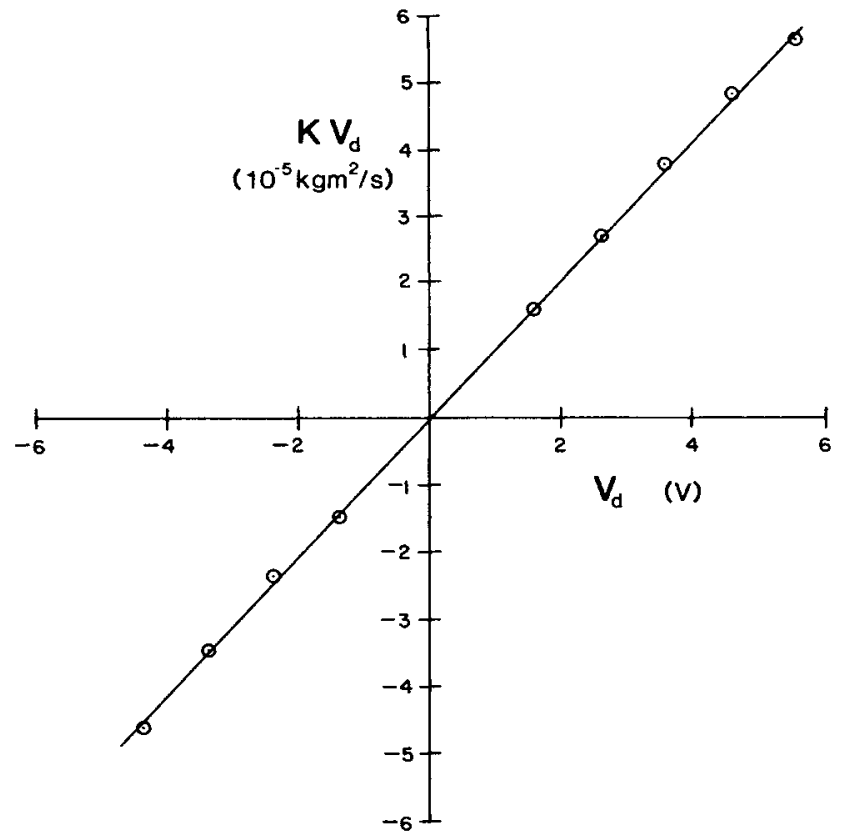

FIG. 14. Measured response of apparatus, with pendulum removed, to drive voltage.

ring magnet and optical encoding disk attached to the axle. In this case, Eq. (1) reduces to

$$
I \frac{d^{2} \theta}{d t^{2}}+K V_{d} \frac{d \theta}{d t}=0
$$

If $V_{d}$ is a constant voltage, then the solution of Eq. (5)

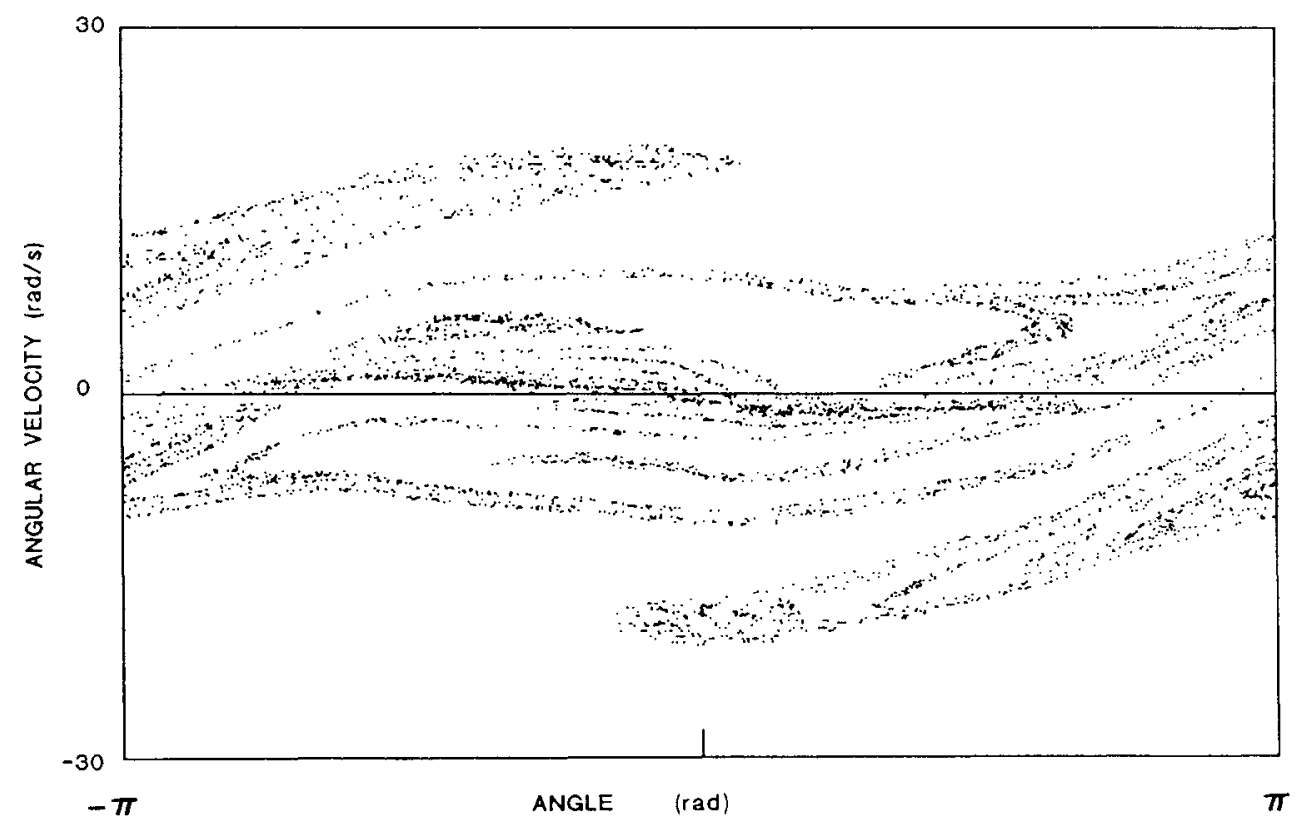

FIG. 15. Experimental Poincaré plot for $Q=18.33, \epsilon=19.8$, and $\Omega=1.80$. Note the similarity to the simulation result shown in Fig. 11 (c). 


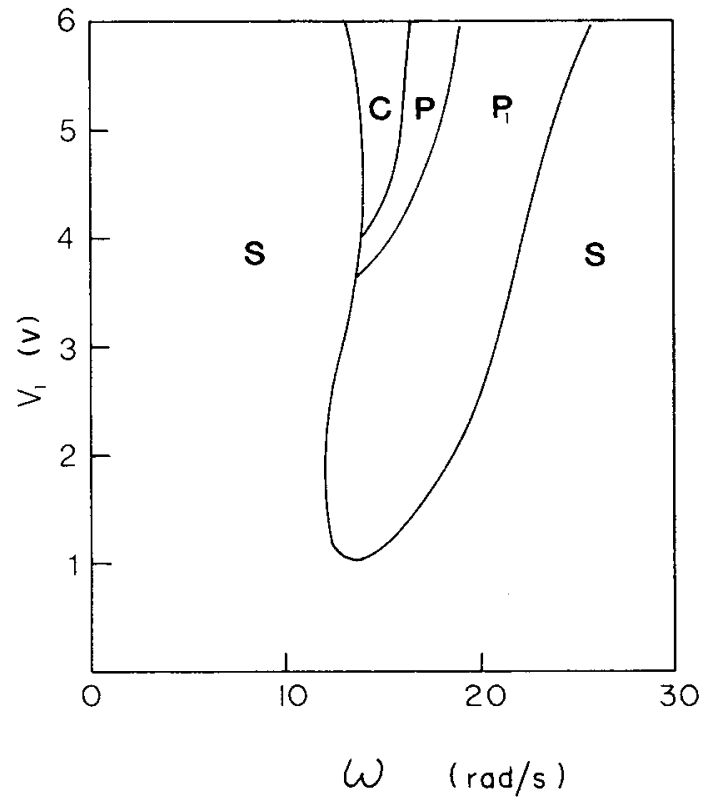

FIG. 16. Experimental state diagram for the parametrically damped pendulum. The regions are labeled as in Fig. 1.

implies $d \theta / d t=(d \theta / d t)_{0} \exp \left(-K V_{d} t / I\right)$. Positive control voltages produce an exponential decay of angular velocity, while negative $V_{d}$ cause an acceleration. Experiments were performed for a series of control voltages of both polarities, and the corresponding time constants $\left(I / K V_{d}\right)$ for each case of acceleration or deceleration were obtained from the spin-up or spin-down data. Since the moment of inertia of the system without the pendulum attached had been determined to be $I=4.718 \times 10^{-6}$ $\mathrm{kg} \mathrm{m}^{2}$, it was then possible to plot $K V_{d}$ versus $V_{d}$, as shown in Fig. 14. The fact that the observed points closely follow a linear relationship verifies that the design does indeed behave according to Eq. (4). From the slope of this figure, we find $K=1.04 \times 10^{-5} \mathrm{~kg} \mathrm{~m}^{2} / \mathrm{s} \mathrm{V}$.

The pendulum will be governed by Eq. (2) provided the drive voltage is chosen to be $V_{d}=V_{0}+V_{1} \sin (\omega t)$, with $V_{0}=A / K$ and $V_{1}=B / K$. In the notation of Eq. (3) $Q=(m g b I)^{1 / 2} / K V_{0}$ and $\epsilon=V_{1} / V_{0}$. As constructed, the system with pendulum attached had a total moment of inertia $I=5.66 \times 10^{-6} \mathrm{~kg} \mathrm{~m}^{2}$, a free oscillation frequency $\omega_{0}=8.42 \mathrm{rad} / \mathrm{s}$, and a value for $m g b$ equal to $4.01 \times 10^{-4}$ $\mathrm{kg} \mathrm{m}^{2} / \mathrm{s}^{2}$. The dc component of the drive voltage was usually fixed at $0.25 \mathrm{~V}$, resulting in a $Q$ of 18.33. The ac amplitude $V_{1}$ was typically set within the range 0 to $6 \mathrm{~V}$, corresponding to $\epsilon$ values from 0 to 24 .

All of the dynamical phenomena described in the preceding section have been observed with the pendulum apparatus. We will not present the many experimentally derived phase plane trajectories that correspond to the various simulation results. Instead, only two representative figures will now be discussed.

Figure 15 is an experimentally observed Poincaré section for $Q=18.33$. The drive frequency was $\omega=15.1$ $\mathrm{rad} / \mathrm{s}(\Omega=1.80)$ and the ac amplitude was $4.95 \mathrm{~V}$ $(\epsilon=19.8)$. This strange attractor agrees very well with the simulation result already presented in Fig. 11(c). In comparing the two figures, recall that the vertical scale in Fig. 11(c) is expressed in normalized time units; to convert to real time, the scale must be multiplied by $\omega_{0}$.

A state diagram for the pendulum was determined by choosing an array of values for $V_{1}$ and $\omega$, and recording the observed motion (stationary, periodic, multiperiodic, or chaotic). The result of this process is shown in Fig. 16. In general, there is agreement with all the features of the simulation state diagram (Fig. 1), although some as yet undetermined experimental errors have distorted the domain boundaries in Fig. 16.

\section{ACKNOWLEDGMENTS}

Financial support was provided by the Natural Sciences and Engineering Research Council of Canada.
${ }^{1}$ Francis C. Moon, Chaotic Vibrations (Wiley, New York, 1987).

${ }^{2}$ R. L. Kautz and R. Monaco, J. Appl. Phys. 57, 875 (1985).

${ }^{3}$ W. C. Kerr, M. B. Williams, A. R. Bishop, K. Fesser, P. S. Lomdahl, and S. E. Trullinger, Z. Phys. B 59, 103 (1985).

${ }^{4}$ D. D'Humieres, M. R. Beasley, B. A. Huberman, and A. Libchaber, Phys. Rev. A 26, 3483 (1982).

${ }^{5}$ A. H. MacDonald and M. Plischke, Phys. Rev. B 27, 201 (1983).

${ }^{6}$ J. A. Blackburn, Yang Zhou-Jing, S. Vik, H. J. T. Smith, and M. A. H. Nerenberg, Physica 26D, 385 (1987).

${ }^{7}$ S. Beckert, U. Schock, C.-D. Schulz, T. Weidlich, and F. Kaiser, Phys. Lett. 107A, 347 (1985).

${ }^{8}$ R. W. Leven, B. Pompe, C. Wilke, and B. P. Koch, Physica 16D, 371 (1985).

${ }^{9}$ B. P. Koch and R. W. Leven, Physica 16D, 1 (1985).

${ }^{10}$ B. P. Koch, R. W. Leven, B. Pompe, and C. Wilke, Phys. Lett. 96A, 219 (1983).
${ }^{1 '}$ R. W. Leven and B. P. Koch, Phys. Lett. 86A, 71 (1981).

${ }^{12}$ K. Inoue, J. Phys. Soc. Jpn. 57, 1226 (1988).

${ }^{13}$ S. W. Shaw and S. Wiggins, Physica 31D, 190 (1988).

${ }^{14}$ F. J. Romeiras and E. Ott, Phys. Rev. A 35, 4404 (1987).

${ }^{15}$ R. A. Nelson and M. G. Olsson, Am. J. Phys. 54, 112 (1986).

${ }^{16}$ P. T. Squire, Am. J. Phys. 54, 984 (1986).

${ }^{17}$ L. Collatz, The Numerical Treatment of Differential Equations (Springer-Verlag, New York, 1966), see pp. 68-78.

${ }^{18}$ P. M. Battelino, C. Grebogi, E. Ott, J. A. Yorke, and E. D. Yorke, Physica 32D, 296 (1988).

${ }^{19}$ C. Grebogi, E. Ott, and J. A. Yorke, Physica 24D, 243 (1987).

${ }^{20}$ A. Davidson and N. F. Pedersen, Phys. Rev. A 36, 2455 (1987).

${ }^{21}$ M. Iansiti, Quing Hu, R. M. Westervelt, and M. Tinkham, Phys. Rev. Lett. 55, 746 (1985).

22 J. A. Blackburn, S. Vik, Wu Binruo, and H. J. T. Smith, Rev. Sci. Instrum. 60, 422 (1989). 